\title{
Efecto del fraccionamiento del nitrógeno en las características agronómicas del trigo
}

\section{Effect of nitrogen fractionation on the agronomic characteristics of wheat}

Victor Araujo ${ }^{1}$, Diosnel Amarilla M. ${ }^{1}$, Milciades Melgarejo ${ }^{1}$, Ever Maidana ${ }^{1}$, Elida Peralta ${ }^{1}$, Miguel Bogado ${ }^{1}$, Rafael Franco ${ }^{1}$, Oscar Silvero ${ }^{1}$, Patricia Colman ${ }^{1}$, Ricardo Arce ${ }^{2}$, Alcides Villalba ${ }^{2}$

\author{
1 Universidad Nacional de Canindeyú, Facultad de Ciencias Agropecuarias y \\ Ambientales. Katueté, Paraguay. \\ 2 Instituto Paraguayo de Tecnología Agraria-IPTA, Campo Experimental Área Cultivo \\ de Trigo. Colonia Yjhovy, Paraguay. \\ Autor de correspondencia: diosnel.amarilla@gmail.com
}

Resumen: El nitrógeno es un elemento muy móvil en el suelo por lo que requiere de aplicación fraccionada en diferentes momentos del crecimiento del trigo para su mejor rendimiento, este trabajo fue realizado con el objetivo de evaluar el efecto de diferentes momentos de aplicación de urea en cultivo de trigo. El experimento se instaló en el Campo Experimental de (IPTA) Yjhovy cuya coordenadas $\left(24^{\circ} 16^{\prime} 26^{\prime \prime} \mathrm{S}\right.$ y $\left.55^{\circ} 01^{\prime} 02^{\prime \prime} 0\right)$. El diseño fue el de bloques completo al azar con 5 tratamientos y 5 bloques, totalizando 25 unidades experimentales, los tratamientos consistieron en la aplicación fraccionada de urea en momento de siembra (MS), 30 días después de siembra (30 DDS) y 60 días después de siembra (60DDS), quedando los tratamientos de la siguiente forma: T1 (Testigo) , T2 (200 $\mathrm{kg} \mathrm{ha}^{-1}$ de N en MS), T3(100kg ha-1 de N en MS y $100 \mathrm{~kg} \mathrm{ha}^{-1}$ a los $\left.60 \mathrm{DDS}\right), \mathrm{T} 4\left(100 \mathrm{~kg} \mathrm{ha}^{-1} \mathrm{de} \mathrm{N}\right.$ a los 30DDS y $100 \mathrm{~kg} \mathrm{ha}^{-1}$ de $\mathrm{N}$ a los 60DDS), T5(66.66 $\mathrm{kg} \mathrm{ha}^{-1}$ de $\mathrm{N}$ en MS, $66.66 \mathrm{~kg} \mathrm{ha}^{-1}$ a los 30DDS y $66.66 \mathrm{~kg} \mathrm{ha}^{-1} 60 \mathrm{DDS}$ ). Las variables evaluadas fueron; altura de planta, cantidad de espigas, longitud de espiga, peso de 1000 granos, rendimientos y peso hectolítrico. Los datos fueron sometidos al ANAVA y al test de Tukey al 5\%. Los tratamientos tuvieron efecto significativo en todas las variables estudiadas, y se obtuvo la máxima eficiencia del fertilizante con el tratamiento T5 que expresó el mejor valor de medias para todas las variables analizadas.

Palabras clave: trigo, fraccionamiento, fertilización, urea, nitrógeno. Abstract: Nitrogen is a very mobile element in the soil, so it requires fractional application at different times of wheat growth for its best 
performance. This work was carried out with the aim of evaluating the effect of different times of application of urea in cultivation of wheat. The experiment was installed in the Yjhovy Experimental Field (IPTA) whose coordinates $\left(24^{\circ} 16^{\prime} 26^{\prime \prime S}\right.$ and $\left.55^{\circ} 01^{\prime} 02^{\prime \prime} \mathrm{W}\right)$. The design was the complete random block design with 5 treatments and 5 blocks, totaling 25 experimental units, the treatments consisted of the fractional application of urea at planting time (MS), 30 days after planting (30 DDS) and 60 days after sowing (60DDS), leaving the treatments as follows: T1 (Control), T2 $200 \mathrm{~kg}$ ha-1 of $\mathrm{N}$ in MS), T3 (100kg ha-1 of N in MS and $100 \mathrm{~kg}$ ha-1 at 60DDS ), T4 (100kg ha- 1 of $\mathrm{N}$ at 30DDS and $100 \mathrm{~kg}$ ha- 1 of $\mathrm{N}$ at $60 \mathrm{DDS}$ ), T5 (66.66 kg ha-1 of $\mathrm{N}$ in MS, 66.66kg ha- 1 at 30DDS and $66.66 \mathrm{~kg}$ ha- $160 \mathrm{DDS}$ ). The evaluated variables were; plant height, number of ears, length of ear, weight of 1000 grains, yields and hectoliter weight. The data were submitted to ANAVA and the $5 \%$ Tukey test. The treatments had a significant effect on all the variables studied, and maximum fertilizer efficiency was obtained with the T5 treatment, which expressed the best mean value for all the variables analyzed.

Keywords: wheat, fractionation, fertilization, urea, nitrogen.

\section{INTRODUCCIÓN}

El cultivo del trigo se encuentra entre los cereales de mayor importancia en el mundo, debido a las propiedades físicas y químicas que posee, principalmente en el gluten, que favorece para producir pan de buen volumen, también es utilizado para la producción en confitería para la producción pastelera y también en la elaboración de sémola y otros derivados.

Sin embargo, para la buena producción de este cultivo se requiere una buena fertilización y condiciones ambientales adecuadas, en este sentido, durante la antesis y hasta la madurez fisiológica, son deseados temperaturas de 16 a $30^{\circ} \mathrm{C}$, a partir de este rango por cada grado centígrado de aumento se produce una disminución de rendimiento de grano de aproximadamente 3 a 4 \% (1).

Con relación a la fertilización son necesarios macro y micro elementos, siendo que para producir $1.000 \mathrm{~kg}$ de granos, el cultivo demanda de $30 \mathrm{~kg}$ de nitrógeno $(\mathrm{N}), 15 \mathrm{~kg}$ de fosforo $(\mathrm{P})$ y $30 \mathrm{~kg}$ de potasio $(\mathrm{K})$.

El nitrógeno $(\mathrm{N})$ constituye el principal factor limitante de la productividad del trigo. Visando la necesidad de $\mathrm{N}$ por parte del cultivo, el uso de la urea como fuente de nitrógeno en trigo es fundamental para sostener e incrementar la productividad de los sistemas de producción, debido a que el 
agregado de $\mathrm{N}$ fomenta la expansión foliar y el macollaje del cultivo, que en general determinan el rendimiento y la calidad del producto(3).

Considerando que los productores de trigo conocen sobre la importancia del abonado nitrogenado para la obtención de altos rendimientos, surge la incógnita relacionada con la cantidad adecuada a ser aplicado y el momento exacto de aplicación en el cultivo. Dosis inferiores al exigido por el cultivo, reducirá el potencial de rendimiento y en dosis superiores ocurrirá una reducción de las ganancias por el gasto innecesario en abonado, perjuicios al ambiente, contaminación del agua superficial y subterráneas ocasionados por lixiviación del nitrato(4).

La aplicación de $(\mathrm{N})$ cerca de la floración reduce rendimiento e incrementa la proteína en los granos y la aplicación post floración induce producir proteínas solubles como la albumina y globulina(5). Las fertilizaciones fraccionadas entre siembra y macollaje son una alternativa para sincronizar la demanda de nitrógeno del cultivo con los aportes del suelo y los fertilizantes, el momento de incorporación efectiva del fertilizante nitrogenado en el suelo condicionan el componente del rendimiento afectado y su eficiencia de uso. La máxima eficiencia se obtiene cuando la aplicación se realiza lo más tarde posible con estadios de crecimiento que permitan una rápida captación( ${ }^{(6)}$.

De tal manera es de gran interés realizar el estudio de los diferentes momentos de aplicación del nitrógeno en forma fraccionada, con el objetivo de determinar el momento de tiempo más adecuado en la etapa vegetativa para la fertilización, ya que el $\mathrm{N}$ es considerado uno de los nutrientes esenciales en el cultivo de trigo, y una deficiencia en una de las diferentes fases de desarrollo, limitaría considerablemente no sólo la producción, sino también la calidad del grano.

\section{MATERIALES Y MÉTODOS}

El ensayo se realizó en el campo experimental (IPTA) de Yjhovy cuyas coordenadas geográficas son $24^{\circ} 16^{\prime} 26^{\prime \prime} \mathrm{S}$ y $55^{\circ} 01^{\prime} 02^{\prime \prime} \mathrm{O}$. El tipo de suelo está clasificado como del orden Oxisol, subgrupo Rhodic y gran grupo Kandiudox, con buen drenaje y pedregosidad nula. En los Oxisoles, las arcillas predominantes son los óxidos hidratados de hierro (Fe) y de aluminio (Al) y la caolinita, a que transfieren al suelo una baja capacidad de intercambio catiónico ${ }^{(7)}$. Para la instalación de la parcela, se recolectaron 10 submuestras de suelo de 0 a $0.20 \mathrm{~m}$ para la determinación de las características químicas y granulometría del suelo, cuyo resultado son los siguientes: $\mathrm{pH}(\mathrm{CaCl} 2)=5.0$; M.O = 31.10 g dm-3; P (Mehlich I) $=33.10 \mathrm{mg} \mathrm{dm}^{-3} ; \mathrm{K}+=1,14 \mathrm{cmol} \mathrm{dm}^{-3} ; \mathrm{Ca}$ $++=4,70 \mathrm{cmolc} \mathrm{dm}^{-3} ; \mathrm{Mg}++=1,60 \mathrm{cmolc} \mathrm{dm}^{-3} ; \mathrm{V}=58,17 \%$; Arena $=30,45 \%$ 
de granulometría; limo $=20,65 \%$ y arcilla $=48,90 \%$ respectivamente. El clima de la región es subtropical, temperaturas y precipitaciones medias anuales de $25^{\circ} \mathrm{C}$, y $1.800 \mathrm{~mm}$, viento predominante del norte. Durante el desarrollo del cultivo se obtuvieron los siguientes datos meteorológicos (Figura1) cuyo resultado fueron proveídos por la estación meteorológica del IPTA Yjhovy ${ }^{(8)}$.

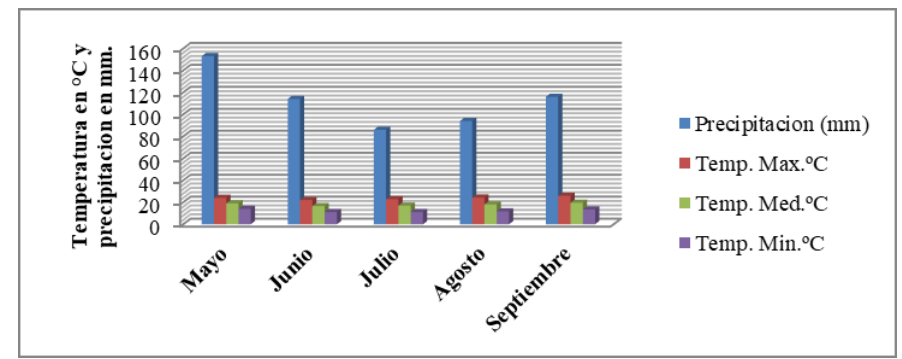

Figura 1: Datos meteorológicos durante el ensayo sobre el cultivo del trigo (Triticum aestivum). Katuete, 2020.

Quince días antes de la siembra se realizó una desecación de las malezas presentes en el sitio con glifosato de $3.0 \mathrm{~L} \mathrm{ha}^{-1}$. El área experimental tuvo una dimensión total de $340.75 \mathrm{~m}^{2}$, las unidades experimentales fueron conformadas por 9 líneas de $5 \mathrm{~m}$ de largo, con una distancia de $1 \mathrm{~m}$ entre cada unidad experimental, el área útil consistió en 5 líneas con $3 \mathrm{~m}$ de largo, totalizando un área de 2,55 $\mathrm{m}^{2}$.

La siembra se realizó de forma manual, con un espaciamiento de $0.17 \mathrm{~m}$ entre surcos y 80 semillas por $m$ lineal a una profundidad de 3 a $5 \mathrm{~cm}$, con una densidad población aproximada de 400 plantas por $\mathrm{m}^{2}$. La variedad utilizada en el experimento fue el COODETEC 150.

Se utilizó el diseño de bloques completo al azar con 5 tratamientos y 5 repeticiones, totalizando 25 unidades experimentales. Los tratamientos consistieron en la aplicación fraccionada y al voleo de la urea como fuente de nitrógeno en el momento de la siembra, a los 30 días y a los 60 días como se detalla en la Tabla 1.

Tabla 1. Dosis de $\mathrm{N}\left(\mathrm{kg} \mathrm{ha}^{-1}\right)$ en el momento de la siembra (MS), 30 días después de la siembra (30DDS) y 60 días después de la siembra (60DDS) en el cultivo del trigo. Katueté, 2020.

\begin{tabular}{llll}
\hline Tratamientos & $\begin{array}{l}\text { Dosis (N) en kg ha-1 } \\
\text { MS }\end{array}$ & $\begin{array}{l}\text { Dosis (N) en kg ha- } \\
\mathbf{1} \text { 30DDS }\end{array}$ & $\begin{array}{l}\text { Dosis (N) } \\
\text { en kg ha- } \\
\mathbf{1} \text { 60DDS }\end{array}$ \\
\hline T1 & & 0 \\
T2 & 0 & 0 & 0 \\
T3 & 200 & 0 & 100 \\
T4 & 100 & 0 & 100 \\
T5 & 0 & 100 & 66,66 \\
\hline
\end{tabular}

Araujo V, Amarilla MD, Melgarejo M, Maidana E, Peralta E, Bogado M, Franco R, Silvero O, Colman P, Arce R, Villalba A. Efecto del fraccionamiento del nitrógeno en las características agronómicas del trigo. 
La aplicación de la urea se realizó en el momento de la siembra en forma manual abriendo surcos en forma paralela a la línea de siembra con un distanciamiento de $5 \mathrm{~cm}$ y una profundidad de $6-8 \mathrm{~cm}$ y la aplicación posterior a la siembra fue realizada en forma manual al voleo para cada tratamiento de forma diferenciada.

El control de malezas se realizó mediante la aplicación de herbicida preemergente y posteriormente en la etapa vegetativa se realizó el control de forma manual, mediante el uso de azada de acuerdo a la necesidad y para el control de plagas y enfermedades se aplicó el insecticida, Imidacloprid 35\% con dosis de 50-60 cc ha-1 y fungicida Tebuconazol 70\% dosis $50 \mathrm{cc} \mathrm{ha}^{-1}$.

La cosecha se realizó después de la maduración fisiológica del grano, con una humedad del $13 \%$, en forma manual cosechando la parcela útil y luego fue trillado.

Las variables evaluadas fueron: altura de la planta, cantidad de espigas, longitud de espigas, peso de 1.000 granos, rendimiento y peso hectolítrico.

El análisis estadístico se realizó a través del análisis de varianza (ANAVA) y la comparación de media con el Test de Tukey al 5\% de probabilidad de error, con el software (SASM-Agri) ${ }^{(9)}$.

\section{RESULTADOS Y DISCUSIÓN}

Todas las variables evaluadas presentaron diferencias significativas entre tratamientos $(p<0.05)$

El tratamiento 5 presento mayor altura de plantas (Tabla 2), estos resultados demuestran un efecto positivo para el desarrollo de la planta al disponer de $\mathrm{N}$ durante todo su desarrollo vegetativo y parte del reproductivo, y concuerda con lo propuesto por Clementelli y Zeballos(10), que la absorción tardía de $\mathrm{N}$ retrasa la maduración al estimular el desarrollo vegetativo, la aplicación de $\mathrm{N}$ en la época adecuada acelera el crecimiento inicial y aumenta la precocidad.

Sin embargo, para la cantidad de espigas, los tratamientos T2, T3 y T5 presentaron mayor cantidad de esta variable en comparación a los demás (Tabla 2). La aplicación de urea en el momento de la siembra tuvo un efecto positivo para la variable cantidad de espiga por $\mathrm{m}^{2}$ en relación a los tratamientos que no se han aplicado urea en el momento de la siembra (T1 y T4), esto pudo deberse al efecto positivo del nitrógeno al inicio del cultivo para el ahijamiento o macollaje de las plantas y así poder aumentado la cantidad de número de espiga por $\mathrm{m}^{2}$, esto concuerda con lo afirmado por Oropesa(11), que el $\mathrm{N}$ afecta a los 3 componentes del rendimiento: espigas por hectáreas, granos por espiga y peso de grano. 
Para la variable longitud de espiga se observaron resultados estadísticamente superiores para los tratamientos T4 y T5, que presentaron mayor longitud de espiga con $7,65 \mathrm{~cm}$ y $7,25 \mathrm{~cm}$ respectivamente, (Tabla 2). Al aplicar urea en la etapa vegetativo y reproductivo, presentó mayor longitud de espiga, lo que favoreció el buen desarrollo vegetativo y reproductivo del trigo ${ }^{(10,11)}$, ya que los cereales tienen requerimiento de nitrógeno desde el crecimiento vegetativo hasta su madurez, sin embargo cualquier deficiencia en la nutrición nitrogenada durante su vegetación se traduce en la reducción de los rendimiento.

Tabla 2. Comparación de medias de los tratamientos evaluados (diferentes momentos de aplicación de N) para las variables altura de plantas (AP - cm), cantidad de espigas (CE - № $\mathrm{m}^{2}$ ) y longitud de espiga ( $\mathrm{LE}$ - $\mathrm{cm}$ ) en en el cultivo de trigo. Katuete, 2020.

\begin{tabular}{llll}
\hline Tratamientos & AP(cm) & CE $\left(\mathbf{m}^{2}\right)$ & LE $(\mathbf{c m})$ \\
\hline (T1)0-0-0 & $71.192 \mathrm{~d}^{*}$ & $429.4 \mathrm{~b}^{*}$ & $6.54 \mathrm{c}^{*}$ \\
(T2)200-0-0 & $75.546 \mathrm{bc}$ & $549 \mathrm{a}$ & $6.79 \mathrm{c}$ \\
(T3)100-0-100 & $78.220 \mathrm{~b}$ & $545.4 \mathrm{a}$ & $6.97 \mathrm{bc}$ \\
(T4)0-100-100 & $73.094 \mathrm{~cd}$ & $439.6 \mathrm{~b}$ & $7.65 \mathrm{a}$ \\
(T5)66,6-66,6-66,6 & $81.674 \mathrm{a}$ & $515.4 \mathrm{a}$ & $7.25 \mathrm{ab}$ \\
\hline
\end{tabular}

* Medias seguidas por la misma letra no difieren estadísticamente por el test de Tukey $5 \%$.

Para las variables peso de 1.000 granos y para el rendimiento del T5 presentó resultados estadísticos superiores en relación a los demás tratamientos, con $40,50 \mathrm{~g}$ y $3.047 \mathrm{~kg} \mathrm{ha}^{-1}$, respectivamente (Tabla 3). Este efecto positivo puede deberse a la aplicación fraccionada de $\mathrm{N}$, lo cual permite la disponibilidad del nutriente en la dosis y el momento correcto, logrando una maxima eficiencia de uso en el sistema de producción, incrementando la productividad, lo cual concuerda con lo mencionado por Ventimiglia y Torrens(12). Ademas de disponibilizar el $\mathrm{N}$ en la etapa vegetativa, la disponibilidad del mismo al inicio de la etapa reproductiva puede permitir suplir la demanda elevada de este nutriente por parte del cultivo y asegurar la fijación de un alto número de flores fértiles, lo cual es determinante para definir el número y el peso de granos por espiga, esto coincide con el manual de guía práctica sobre trigo(13) en el cual menciona que la aplicación debe ser realizada a fin de macollamiento o durante el periodo crítico de formación de los granos entre 20 días y hasta 7 días después de floración.

Mientras que para la variable peso hectolitrico, el cual es uno de los parámetros que mide la calidad física del grano del trigo, los tratamientos T5 y T3 se destacaron estadísticamente en relación a los demás tratamientos, 


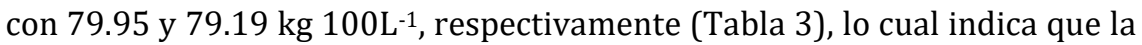
disponibilidad de $\mathrm{N}$ tanto al inicio de la fase vegetativa como de la fase reproductiva son fundamentales para la obtención de la calidad comercial e industrial del trigo. Este resultado coincide con lo propuesto por García ${ }^{(14)}$ en el cual menciona que la fertilización con nitrógeno $\mathrm{N}$ mejora el rendimiento, el porcentaje de proteína y otros parámetros de calidad comercial e industrial.

Tabla 3. Comparación de medias de los tratamientos evaluados (diferentes momentos de aplicación de N) para las variables peso de mil granos (PMG - g),

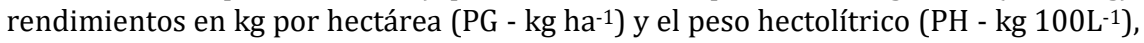
en el cultivo del trigo. Katuete, 2020.

\begin{tabular}{llll}
\hline Tratamientos & PMG & PG & PH \\
\hline (T1) 0-0-0 & $32.61 \mathrm{c}^{*}$ & $2257.2 \mathrm{~d}^{*}$ & $75.08 \mathrm{~d}^{*}$ \\
(T2) 200-0-0 & $33.63 \mathrm{c}$ & $2569.2 \mathrm{bc}$ & $77.15 \mathrm{c}$ \\
(T3) $100-0-100$ & $37.71 \mathrm{~b}$ & $2745.4 \mathrm{~b}$ & $79.19 \mathrm{ab}$ \\
(T4) 0-100-100 & $34.36 \mathrm{c}$ & $2387.6 \mathrm{~cd}$ & $78.49 \mathrm{~b}$ \\
(T5) 66,6-66,6-66,6 & $40.50 \mathrm{a}$ & $3047 \mathrm{a}$ & $79.95 \mathrm{a}$ \\
\hline
\end{tabular}

* Medias seguidas por la misma letra no difieren estadísticamente por el test de Tukey $5 \%$.

\section{CONCLUSIÓN}

La aplicación fraccionada del $\mathrm{N}$ en los diferentes momentos de tiempo en la etapa vegetativa del cultivo de trigo, indujo un mayor crecimiento de la planta, mejor desarrollo de espiga y aumento del rendimiento y la calidad de granos del trigo.

\section{AGRADECIMIENTO}

A la Universidad Nacional de Canindeyú (UNICAN) y a la Facultad de Ciencias Agropecuarias y Ambientales (FCAA) por los recursos brindados para la realización del trabajo, y al Instituto Paraguayo de Tecnología Agraria (IPTA) por el espacio cedido para la instalación del experimento.

\section{REFERENCIAS BIBLIOGRÁFICAS}

1. Kohli M, Viedma L, Cubilla LE. Guía para la producción de trigo. Manuel del Productor; 2010.

2. Melgar JR. Compatibilizar la óptima nutrición nitrogenada con una alta eficiencia de uso del n. Buenos Aire: INTA; 2008. 
3. Perdomo C, Barbazán M. Areas de suelo y agua, Nitrógeno, Universidad de la República Facultad de Agronomía; 2004 [consultado el 18 de mayo de 2019].

Disponible

en: www.fagro.edu.py.uy/fertilidad/publica/Tomo\%20N.pdf

4. Lajús et. al. Rendimento de trigo (Triticum aestivum L.) sob diferentes densidades de semeadura e doses de nitrogênio. In: III Seminário Integrado: Ensino, Pesquisa e Extensão, 2011, Chapecó. Ética e Ciência nas Diferentes Dimensões da Educação Superior; 2011.

5. Divito G, García F. (Ed.). Manual del cultivo de trigo. International Plant Nutrition Institute (IPNI); 2012. Disponible en: http://lacs.ipni.net/ipniweb/region/lacs.nsf/0/52054262ADE3411803 2581BF008086C1/\$FILE/Resumen\%20Manual\%20Trigo.pdf

6. Díaz Zorita M. Efecto de dos momentos de aplicación de urea sobre la producción de grano de trigo en drabble (Buenos Aires, Argentina). Ciencia del Suelo; 2000; p 125.

7. Ferreira H, Burian PL. Geografía del Paraguay. Asunción, Paraguay: El Lector; 2002.

8. Climate-Data. Climate-Data.Org. [consultado el 15 de abril de 2019]. Disponible en: https/es.climate-data.org

9. Canteri MG, Althaus RA, Virgens Filho JS, Giglioti EA, Godoy CV. SASM Agri : Sistema para análise e separação de médias em experimentos agrícolas pelos métodos Scoft - Knott, Tukey e Duncan. Revista Brasileira de Agrocomputação. 2001.

10. Clementelli A, Zeballos R. Fertilización Nitrogenada Mineral y Orgánica en el Cultivo de Trigo. 2009.

11. Oropesa JP. Efecto de la fertilización nitrogenada sobre rendimiento y calidad en trigo y cebada [en línea]. Trabajo Final de Ingeniería en Producción Agropecuaria. Facultad de Ciencias Agrarias. Universidad Católica Argentina. 2012. Disponible en: http://bibliotecadigital.uca.edu.ar/repositorio/tesis/efecto-fertilizacionnitrogenada.pdf

12. Ventimiglia L, Torrens L. Mejores prácticas de manejo de la fertilización de trigo: Dos experiencias de INTA 9 de Julio - Buenos Aires, Argentina; 2013. [consultado 5 de febrero de 2020]. Disponible en: http://www.ipni.net/publication/ialacs.nsf/0/C637ECC931E69D9C85257B340054D540/\$FILE/8.pdf

13. IPTA, CAPECO, INBIO. Guía práctica para el manejo y la producción de trigo; 2012. Disponible en: https://docplayer.es/59664817-Guiapractica-para-el-manejo-y-la-produccion-de-trigo-fortalecimiento-de-lainvestigacion-y-difusion-del-cultivo-de-trigo-en-el-paraguay.html

14. García E. Manejo del cultivo de trigo; 2012. 